\title{
Interactive Visual Facets to Support Fluid Exploratory Search
}

\author{
He, Chen
}

ACM

2021-11-27

He , C , Micallef , L , Serim , B , Vuong , T T , Ruotsalo , T \& Jacucci , G 2021, Interactive Visual Facets to Support Fluid Exploratory Search . in K Klein, M Burch, D Limberger \& M Trapp (eds), VINCI 2021: The 14th International Symposium on Visual Information Communication and Interaction . ACM , International Symposium on Visual Information Communication and Interaction, Potsdam , Germany , 06/09/2021 . https://doi.org/10.1145/3481549.3481565

http://hdl.handle.net/10138/338796

https://doi.org/10.1145/3481549.3481565

cc_by

acceptedVersion

Downloaded from Helda, University of Helsinki institutional repository.

This is an electronic reprint of the original article.

This reprint may differ from the original in pagination and typographic detail.

Please cite the original version. 


\section{Interactive Visual Facets to Support Fluid Exploratory Search}

\author{
Chen $\mathrm{He}^{*}$ \\ chen.he@helsinki.fi \\ University of Helsinki \\ Finland \\ Tung Vuong \\ University of Helsinki \\ Finland \\ vuong@cs.helsinki.fi
}

\author{
Luana Micallef \\ lm@di.ku.dk \\ University of Copenhagen \\ Denmark \\ Tuukka Ruotsalo \\ University of Helsinki \\ Finland \\ tuukka.ruotsalo@helsinki.fi
}

\author{
Barış Serim \\ University of Helsinki \\ Finland \\ baris.serim@helsinki.fi \\ Giulio Jacucci* \\ University of Helsinki \\ Finland \\ giulio.jacucci@helsinki.fi
}

\begin{abstract}
Exploratory search starts with ill-defined goals and involves browsing, learning, and formulating new targets for search. To fluidly support such dynamic search behaviours, we focus on devising interactive visual facets (IVF), visualising information facets to support user comprehension and control of the information space. To do this, we reviewed existing faceted search interfaces and derived two design requirements (DR) that have not been fully addressed to support fluid interactions in exploratory search. We then exemplified the requirements through devising an IVF tool, which coordinates a linear and a categorical facet representing the distribution and summarisation of items, respectively, and providing context for faceted exploration (DR1). To support rapid transitions between search criteria (DR2), the tool introduces a novel design concept of using facets to select items without filtering the item space. Particularly, we propose a filter-swipe technique that enables users to drag a categorical facet value sequentially over linear facet bars to view the items in the intersection of the two facets along with the categorical facet dynamically summarizing the items in the interaction. A user study of 11 participants with realistic email search tasks shows that dynamic suggestions through the timeline navigation can help discover useful suggestions for search; the novel design concept was favoured over using facet values as filters. Based on these practices, we derive IVF design implications for fluid, exploratory searches.
\end{abstract}

\section{CCS CONCEPTS}

-Information systems $\rightarrow$ Search interfaces; $\bullet$ Human-centered computing $\rightarrow$ Interaction design.

\section{KEYWORDS}

exploratory search, information facets, fluid interaction

\section{ACM Reference Format:}

Chen He, Luana Micallef, BarışS Serim, Tung Vuong, Tuukka Ruotsalo, and Giulio Jacucci. 2021. Interactive Visual Facets to Support Fluid Exploratory Search. In Proceedings of ACM Conference (Conference'17). ACM, New York, NY, USA, 9 pages. https://doi.org/10.1145/nnnnnnn.nnnnnnn

${ }^{*}$ Corresponding authors

Conference'17, fuly 2017, Washington, DC, USA

2021. ACM ISBN 978-x-xxxx-xxxx-x/YY/MM. . \$15.00

https://doi.org/10.1145/nnnnnnn.nnnnnnn

\section{INTRODUCTION}

Information search is an essential activity that we carry out on a daily basis. Modern browsers have therefore seamlessly integrated search functionalities by enabling users to simply input search queries in the top address bar and retrieve a ranked list of relevant links. However, unlike with simple lookup searches, users often search with ambiguous information needs or uncertain goals [2]. For instance, when looking for related literature, researchers often start with vague, general queries; when searching for an email, users typically do not have enough memory clues to do a simple lookup search. In such situations, users dynamically modify their search directions as they gain further knowledge of the search space [41, 42]. Prior work termed this type of search as exploratory search $[28,36]$ to indicate its learning, investigative nature.

With exploratory search, a query box plus a ranked list of results provide limited support to users' evolving information needs. Advanced visual interfaces are required to fluidly support the iterative and incremental process. Wildemuth and Freund [42] identified a set of characteristics of exploratory search tasks based on literature review. Exploratory search tasks focus on learning and investigative search goals; they are open-ended, dynamic, and motivated by ill-defined problems [41, 42], which implies the dynamism of user search behaviours. How to design visual interfaces to fluidly support exploratory search is our main research question.

Facets, composed of orthogonal sets of categories [18], can organise information into meaningful groups [41, 42]. Studies on search interfaces with information facets show that users use facets more frequently for exploratory search tasks [30,31]; the use of information facets is essential to support both the expansion and refinement of search queries [41, 42]. Recent work on faceted search puts more efforts on algorithm development and user evaluation [30]. For instance, techniques have been developed to generate nested facets from ontologies [46] and to predict facets of interest based on user interactions [4]. However, interactive visualisation design is also essential in supporting faceted exploration [26].

From a human-computer interaction point of view, there are many benefits of using facets to support fluid exploratory searches. First, facets can serve as entry points for search, which is particularly beneficial when users start with no specific queries in mind [31]. Second, facets can provide an overview of the search space, which avoids users getting lost in the search process [27, 31]. Third, facets can be used as guidance on next relevant directions to explore and avoid zero-hit queries [27]. 
Therefore, our goal is to use information facets to address the challenge of supporting fluid interactions in exploratory search. To do this, we first review prior research on faceted search interfaces and identify their shortcomings in supporting fluid search behaviours. We then propose the concept of interactive visual facets (IVF) and two design requirements, i.e. providing contextual information for faceted exploration and using facets to support rapid transitions between search criteria, which have not been fully addressed by existing work. We exemplify the two requirements through devising an IVF tool. The tool coordinates a linear and a categorical facet and introduces a novel design concept - using facet values to select and inspect items without filtering the item space - to support flexible and dynamic search attempts without losing the exploration context. Particularly, users can drag a categorical facet value sequentially over linear facet bars to view the items in the intersection of the two facet values. Meanwhile, categorical facet changes dynamically to summarize the items in the intersection. To demonstrate the fluidity and usefulness of the features in supporting exploratory search, we present a use case and a user study with emails. The use case illustrates how the features of the tool support email finding. The user study with realistic email search tasks shows that the feature of using facets to select items without filtering the item space was favoured over using facets as filters. Finally, we discuss design implications based on our practices to provide guidance on how to effectively design IVF for fluid exploratory searches in the future.

\section{RELATED WORK}

One of the earliest faceted search interfaces, Flamenco [18], lists hierarchically faceted metadata to guide users toward possible choices. Subsequent faceted search interfaces extended on it from two aspects by 1) visualising facets to aid user comprehension of the information space (e.g. [10, 12, 37, 45]), and 2) taking facets as user-manipulable objects to support information exploration (e.g. [13, 20, 37, 44, 47]).

Apart from listing faceted keywords for exploration, facets are frequently visualised in coordinated views where each view depicts one type of facet, such as chronological and geographical views of the search space; e.g. see [10, 12, 35, 37, 45]. Brushing and linking techniques are used in these cases to support exploration of item distribution in various views. For instance, VisGets [10] coordinates temporal, spatial, and topical views of items to enable users to visually formulate queries from the three aspects in parallel. Likewise, the Visual Backchannel interface coordinates topics, people, time periods, etc. to visualise the evolution of content over time and uses linked brushing and cross filtering for exploration [12].

On the other hand, individual items can be directly drawn upon a facet layer using zooming and panning for item inspection; e.g. see $[11,16,29,40]$. In this case, only one facet is visualised at one time. Themail [40] displays word lists along a timeline to show the evolution of user conversation with an individual. Faceted selection and filtering are supported for detailed inspection. Likewise, Mauro et al. [29] arrange items categorised by colour on a map. Items could be filtered by facets using checkboxes, treemaps, or sunburst views.

To facilitate user exploration, existing visualisations generally support Boolean queries (e.g. [29, 37, 45, 47]) or weight adjustment of facet values (e.g. [3, 6, 7, 20, 22]). For Boolean queries, intra-facet selections filter items through a logical OR operation on the selected values whereas inter-facet selections create a logical AND operation for filtering. For instance, PivotSlice [47] enables data slices in a matrix view through dragging and dropping numeric or categorical facet values to two perpendicular axes. The two query axes support flexible Boolean combination of queries, which results in a logical OR operation on values in the same facet panel of an axis and a logical AND operation over the corresponding panels of the two axes.

Rather than simply using facets to filter results, SearchLens [3] allows users to personalise search results by adjusting the importance of facet values. In uRank, users can reorder search results according to their relevancy to weighted keywords [7-9]. An improved version of uRank incorporated social aspects where users weigh a hybrid model to rank results $[5,6]$.

However, selection or weight adjustment of facet values often results in drastic layout changes of the item space, which inhibits rapid transitions between search criteria. For instance, PivotPaths visualises facet values in nodes and allows pivot operations to transform node layouts to support, such as, single-facet querying and double-facet comparison [13]. Similarly, Fluid Views uses dual layers to position items on top of a context map, such as a timeline or a topic overview [11]. Semantic zooming allows transitions between item overview and details for inspection.

To better support rapid transitions between search criteria, query previews $[32,33]$ are used to put users in the current search context and avoid dramatic layout changes before users are certain of their next search steps. Existing faceted search interfaces incorporate query previews in two ways by showing the number of results related to individual facet values $[18,23,35,37]$ or by highlighting relevant facet values and result items to an attempted query [10, 12, 45]. For instance, FacetScape enables users to view the changes of the number of results before selecting or deselecting facet values. MSpace [44] allows users to arrange facets hierarchically in columns, from left to right, and provides item previews when hovering over facet values to assist in choice-making. This low-cost technique of using hovering interactions to trigger query previews has also been widely used in coordinated views. As an example, VisGets [10] allows users to hover over bars in temporal charts, region circles in geographic views, or topical keywords to see the distribution of related items in the other unhovered views.

The low discontinuity between displayed information invoked by query previews facilitates low-cost, rapid transitions between different queries. However, more preview techniques could be devised to better leverage the advantage.

\section{DEFINITION AND DESIGN REQUIREMENTS}

As elaborated in Section 2, existing faceted search interfaces expanded on information facets in two directions, incorporating visualisation and interaction techniques. Thus, we propose to name information facets as interactive visual facets (IVF) for the purpose of devising faceted interfaces to assist search. We define IVF in exploratory search as visualising information facets to support user comprehension and control of the information space. The goal of this research is to devise IVF to support fluid exploratory searches.

Elmqvist et al. [14] characterised fluidity in information visualisation as smooth interaction, responsive graphics, and conscientious user experience. To support fluid exploratory search, Klouche et 
al. [21] proposed to use entity affordances to devise interaction techniques. Entities can yield other relevant entities to facilitate exploration; entities can be organised to support pattern recognition; and entities can be shared to assist in collaboration (e.g. [1]). Kules and Shneiderman [24] proposed a set of design guidelines of using categorised facets for exploratory search. Shneiderman et al. [38] proposed a four-phase framework for text searches that focused on the iterations of query refinement. However, exploratory searches usually go beyond search queries and involve other types of interactions, such as navigation and browsing. The Flamenco project suggested integrating a direct search method and browsing to enable smooth transitions from one search direction to the next, without users feeling lost or stuck [18].

With previous endeavours in mind, we derive two design requirements that have not been fully addressed so far to drive the design of a fluid, exploratory search interface:

R1: Providing contextual information for faceted exploration. Contextual information can avoid users feeling disconnected from or lost in their search experience [13,18]. Context conveys about the time and space of users' exploration. Time-related context informs users of the exploration process. For instance, which items have been retrieved; which are newly retrieved as results. As Jankun-Kelly et al. [19] stated, a visualisation that does not communicate the states of user visits could go inefficient in assisting data exploration. A review of prior work in Section 2 suggests that this aspect of the requirement was often neglected. Space-related context informs users about the current search space. As stated in Section 1, visualising facets is beneficial in providing an overview of the search space. For example, to guide publication exploration, the facets of related authors and keywords shown in PivotPath depict context about current search queries [13]. Interactions between facets, such as brushing and linking among facet views, can reveal additional relationships about the search space.

R2: Using facets to support rapid transitions between search criteria. During exploratory search, users would modify information needs with the accumulation of knowledge [42], thus user search actions can be tentative. A fluid interface should provide easy transitions between user search attempts with low cognitive or mechanical costs. Query previews can serve this purpose, but existing applications are limited to preview the number of relevant results to facets or highlight relevant facet values under hover (Section 2). Advanced query preview techniques could be devised to better address this requirement.

\section{THE IVF TOOL}

We present a design of IVF addressing the two design requirements to support fluid, exploratory search. Figure 1 shows an overview of the tool implemented with email data. The interface comprises an item snippet view, a facet space, and a query field. This spatial separation provides multiple entry points for search, including item detail exploration, faceted navigation, and query searches. The item snippet view shows snippets of selected items as a stack - newly selected items replace older ones to support skimming [24]. In the case of emails, the snippet view contains the sender, the title, the beginning of the email, and the date. Each row has a coloured dot that represents its freshness through saturation. More saturated colours indicate more recently selected items, which corresponds to the colour of the dots in the linear facet (Figure 2(b)). Clicking on a snippet item opens a new window that shows its details.

The facet space coordinates a linear facet (time in this case) and a categorical facet, such as contact and keyword suggestions. The linear facet area shows the distribution of items over time. A queue of dots in each bar represents a list of items in a specified time period. The range of time for each bar is adjusted automatically according to the number of items over that period, avoiding item overflow. The linear facet view is scalable to display varied numbers of items from the cohort, as it arranges linear spans into pages once it cannot hold all items in one page.

The categorical facet, in the case of emails, consists of keyword and contact suggestions. We extract keywords within the body and subject fields of emails by computing the term frequency-inverse document frequency (TF-IDF) [34] of the keywords and selecting the highest scoring keywords. The keywords are generated by combining words within the same noun phrases detected using dependency parsing [39]. Contacts are extracted from the sender, the recipient, and the carbon-copy field.

The query field allows for typed queries and for dragging a categorical facet value as a query filter. A typed query will be broken down into individual words if it is composed of more than one word. Multiple queries are joined by AND relations, i.e. only text items containing all of the queried words qualify to be displayed. Users can click on a queried word displayed next to the input box to remove the query. When a query is selected or deselected, the tool will update the facets to describe a set of items satisfying the existing queries. In this way, the facets guide users toward the next possible searches and make sure user-dragged queries will not result in an empty result set, i.e. avoid dead ends.

\subsection{R1: Providing contextual information for faceted exploration}

Initially, the most recent items in the timeline view are selected by default. The number of selected items depends on the height of the snippet display, avoiding scrolling. Users can select items by clicking on the dots in the linear facet or scrolling to sequentially select items in the linear bar. The selected dots are shown in purple with varying saturations; more saturated colours represent more recently selected items (Figure 2(b)), which corresponds to the dots in the snippet rows. For the older items that have been pushed out of the stack of the snippet display, dots in the linear facet will turn grey to indicate they have been viewed (Figure 2(c)). Visual encodings of the dots indicate the states of items (i.e. if they are selected, have not or have been selected), and inform users about the exploration process (time-related context of R1).

Mousing-over can trigger the interactions between the linear and categorical facets to support exploration in space-related context. Initially, the categorical facet, contact and keyword suggestions in the case of emails, shows the summarisation of the items in the current span of the linear facet. Hovering over a potential query, which can be a contact or a keyword, highlights the relevant dots in the linear facet as well as in the snippet view with various visual encodings. A line affords a contact relation where the orientation of the sender and co-recipient lines adheres to the general chronological orientation. 


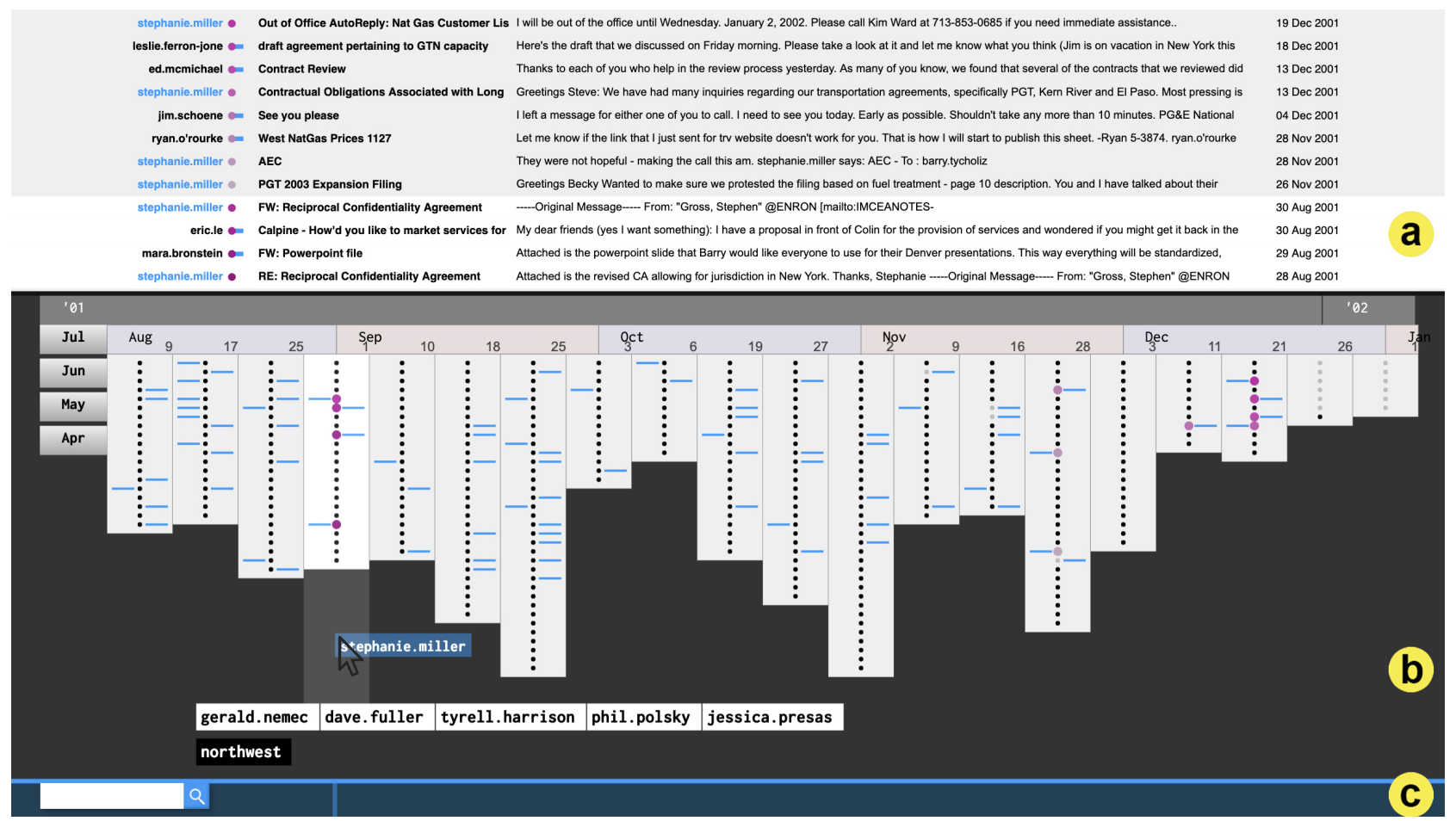

Figure 1: Overview of the IVF tool visualising 400 emails: (a) an email snippet display, (b) time as a linear facet and contact and keyword suggestions as categorical facets, and (c) a query field. A contact, "stephanie.miller", is dragged onto a linear facet bar, so items in the intersection of the two facet values are selected and shown in the snippet view and the categorical facets suggest entities relating to the highlighted items. In this case, highlighted items are emails in this bar that were sent by or carbon-copied to this contact. The snippet display highlights the relevant emails with a white background colour. The categorical facet area suggests contacts and keywords dedicated to these items. If the user find the categorical facet value relevant, s/he can drop it into the query field to filter the items; otherwise, s/he can just release it to end this search attempt. This action is called filter-swipe and supports tentative search actions (R2). Data from the publicly available Enron email corpus [43] are shown.

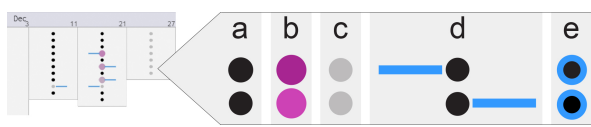

Figure 2: Various states of the dots inform users about the exploration process (R1). Each dot represents an item in the collection. (a) Dots that have not been selected yet; (b) dots under selection. The more saturated colour represents the more recently selected items; (c) dots that have been selected and pushed out of the stack of the snippet view; (d) and (e) the blue marks indicate relationships that can be visually overlaid with the other three states. They depict item relations to the currently focused categorical facet value; in the case of emails, (d) affords contact relations where the left-side line indicates a sender relation and the right-side line denotes a co-recipient relation; (e) denotes keyword relations.

The left-side line indicates a sender relation and the right-side line denotes a co-recipient relation (Figure 2(d)). A circle around a dot depicts a keyword relation (Figure 2(e)). This puts a categorical facet value, such as a topic, in the context of the linear facet (R1), such as time. Users can see, for instance, how the frequency of the topic varies in time through the topic distribution over a timeline. Hovering over a linear facet bar, indicated by the white background of the bar, converts contact and keyword facets to summarise the items in the bar. The dynamism of the summarisations enables the user to assess the next relevant queries in the context of the overall content $(R 1)$. For example, it allows the user to detect special topics in a certain time period among the topics over different time spans. If there are dots being selected in the highlighted bar (i.e. dots in purple), the items of the selected dots in the snippet view are highlighted with a white background colour as well. Mousing over the row of a dot is indicated by a bright blue background, as is the corresponding item in the snippet view.

\subsection{R2: Using facets to support rapid transitions between search criteria}

To support rapid transitions between search attempts, we propose to use facet values to select items for inspection without filtering the item space. Users can click on a categorical facet value for quick item selection. Selected items will be colour-coded in the linear facet, i.e. purple dots with varied levels of saturation, and shown in the snippet view. If this evokes too many items to fit into the snippet display, more recent items will be selected. This design allows users to flexibly inspect items of interest from various perspectives while maintaining the present exploration context. 


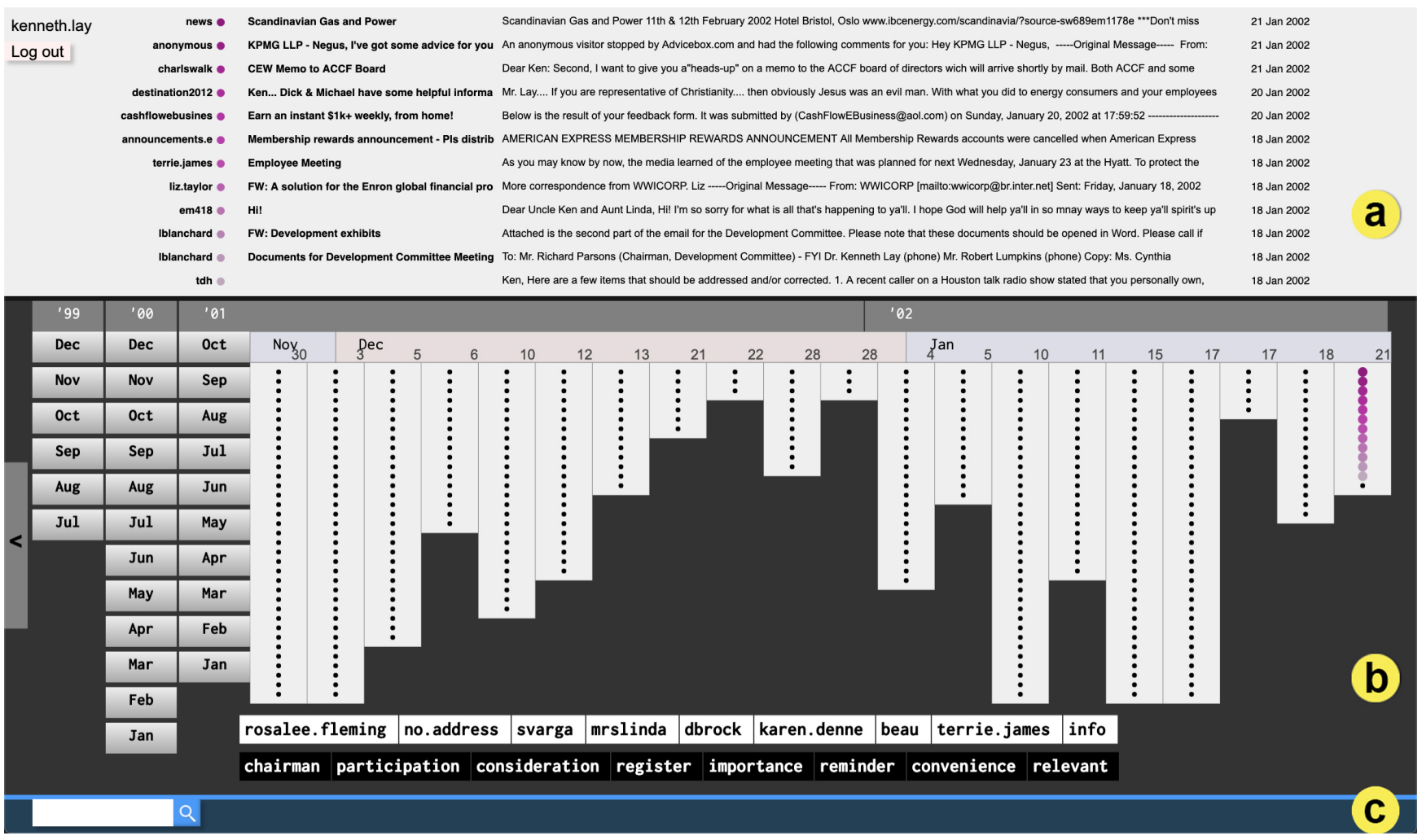

(a) The IVF tool loaded with 2,499 emails for email finding: (a) a snippet view with each row showing one selected email represented by the sender, the title, the beginning of the content, and the date; (b) a facet space with time as a linear facet and contacts and keywords as categorical facets; (c) a query field that supports typed queries as well as dragging categorical facet values as queries.

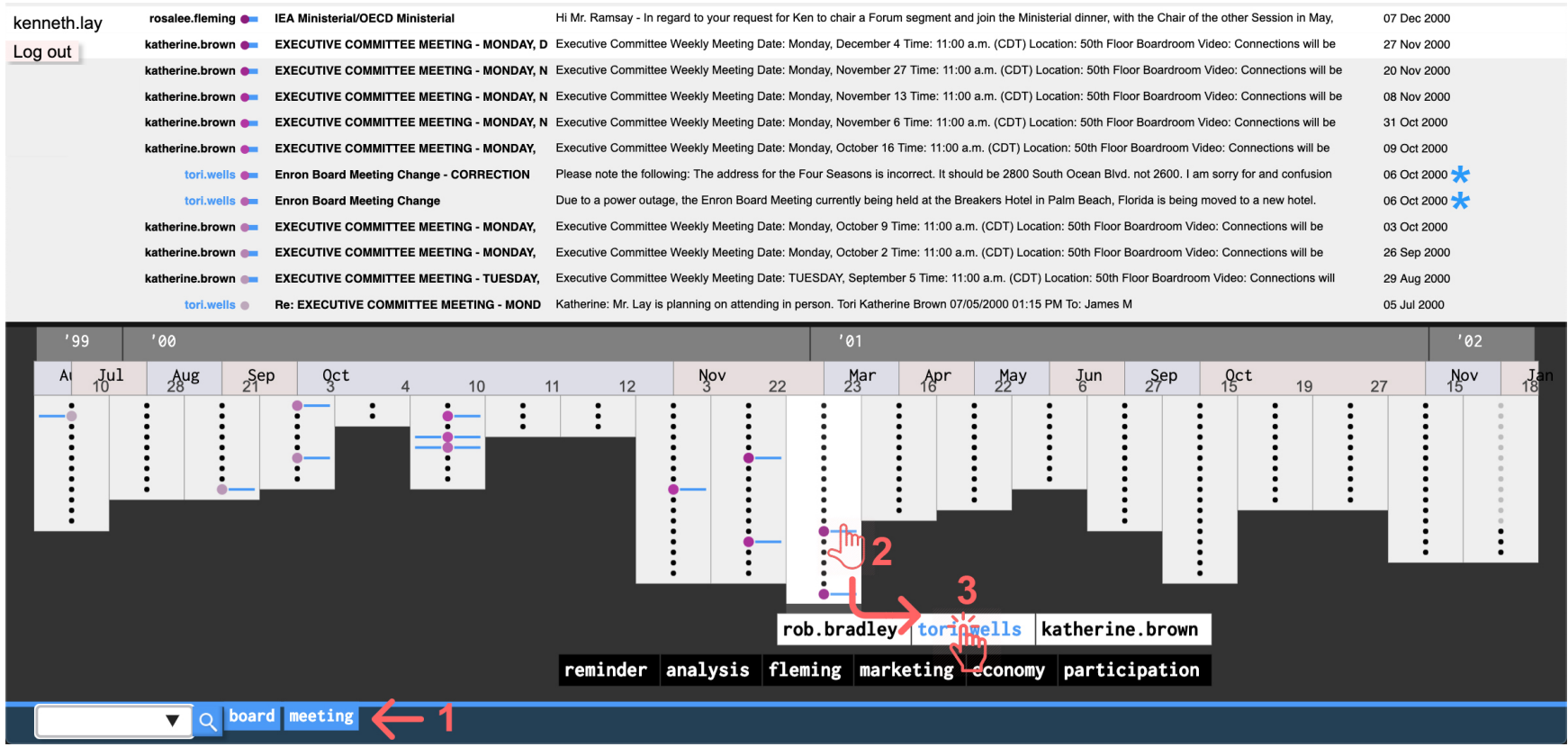

(b) After filtering the emails by queries of "board" and "meeting" (1), Kenneth clicks on the contact "tori.wells" (3) accessed through hovering over the first column of the year 2001 (2) for quick item selection. Selected emails are shown in reverse chronological order in the snippet view and represented by purple dots. Emails marked with ${ }^{*}$ are the target emails.

Figure 3: An email finding scenario. 
The concept of using facets to select items without filtering the item space is further reflected in the design of a novel filter-swipe technique that enables dynamic query previews by flexibly combining two facet values. The user can drag a categorical facet value over linear facet bars sequentially, and as a result, the categorical facet will dynamically show facet values relating to the items in the intersection of the current linear bar and the dragged facet value, and the snippet view will display selected items in the intersection (Figure 1). If the user finds the dragged facet value relevant, he or she can put it in the query field to filter the item space. Otherwise, the user can just release the object to quit this tentative search action and grab another categorical facet value to filter-swipe. The mouse interactions of the filter-swipe technique also trigger the mouse-over and click effects on the categorical facet value. That is, the relevant dots in the linear facet are highlighted with various visual encodings and the most recent items are selected when the user starts to drag the facet value. Overall, the filter-swipe technique enables flexible combinations of a linear and a categorical facet value for item inspection and features rapid transitions between search attempts (R2). See a video demonstration of the IVF tool at https://youtu.be/v0tUAxPjqfg.

\section{USE CASE}

Email, as a daily communication method, requires easy and efficient management. Looking for an email from a large collection of emails is a common yet burdensome task. To demonstrate how the features of the tool address the design requirements and support email finding, we loaded 2,499 emails from the Enron corpus [43] into the IVF tool. See Section 4 for a detailed explanation how the tool functions. We discuss how the IVF tool could help email finding through the following scenario:

Kenneth opens the IVF tool with all his emails (Figure 3a) and wants to find an email about the address of a board meeting happened around early 2001 as he remembers. He types "board meeting" in the query box to filter the emails. The resulting emails range from late 1999 to early 2002 (Figure 3b). He hovers his mouse over the columns around early 2001 and a suggested contact "tori.wells" reminds him of this person's appearance in the meeting. Then he clicked on the contact to quickly select relevant emails. The emails with the address information of the board meeting appeared in the snippet view, which turn out to come in October 2000 (emails marked with * in Figure $3 \mathrm{~b}$ ). In this case, navigating through the linear facet helps the discovery of a familiar contact and further leads to the right email using quick item selection.

\section{USER STUDY}

To understand how people use the IVF tool's features in practical exploratory search scenarios, we conducted a user study involving realistic email search tasks. Locating a specific email in a personal email box with limited memory cues is difficult. Research shows that memories may be organised by episodes, such as the location and relative time of an event [15]. We pondered that user interaction with the tool's facets could assist users' memories and widen search opportunities. In this study, we investigated how users interacted with the coordinated facets to locate a specific email by analysing user interaction data and users' perception on how helpful facets were for email finding through questionnaires.

\subsection{Participants}

We recruited 11 participants from two large universities (6 graduate students, 4 postdoctoral researchers, and 1 administrative staff). A pre-screening questionnaire was administered to ensure that the participants performed email searches at least three times a week and that their email accounts contained more than 300 emails. Four participated the study with their personal email accounts (which they also used for work and study related correspondence) and seven participated with their institutional accounts. All participants were fluent in English and most of their email correspondence was in English.

\subsection{Procedure}

To address privacy issues related to personal data, we adopted a diarykeeping method from Elsweiler et al. [15] to collect email search tasks. To increase the difficulty of the tasks, we carried out the email search experiment 90 days after the diary study was completed.

6.2.1 Diary study. The participants were asked to keep an online diary for 30 days to record all instances in which they had to find an email during this period. We defined the scope of finding as all types of search actions, including typed queries. In total, we collected 127 diary entries (4-18 entries from each participant). We excluded 26 of these entries because (1) they were repeated entries; (2) they did not refer to a certain target email, but rather described cases in which the participant wanted to make sure that there were no emails with specific information; or (3) the target email was in the sent box. This left us with 101 entries, which we then used as email search tasks related to the participant's inboxes.

6.2.2 Lab experiment. The study started with a training session in which each participant completed 4 training tasks that involved finding emails in an unfamiliar inbox, an employee account from the Enron corpus [43], using various combinations of available information, such as sender, topic, co-recipient, and date. Then, we extracted emails within a recent 2-year span from participants' inboxes to the IVF tool. Participants were asked to find the emails mentioned in their diaries using a desktop computer with a 24 -inch monitor. The tasks were introduced in random order and in the form of task cards. Each task card included a diary entry the participant had previously written and a questionnaire. There were no time constraints for executing the tasks. The participants indicated task completion either by opening an email and confirming it as the correct email (success) or by clicking on the "Give up" button on the screen (failure). Participants could skip a task if the task definition written in the diary was too vague or if the specific email was not included in the participant's inbox anymore. An experimenter was present during the study to make sure the procedure was followed and answer any technical questions.

\subsection{Data collection}

During the search tasks, we logged all user interactions and the state of the interface at those moments including filtering criteria, suggestions, and the number and distribution of emails in the timeline interface. To maintain privacy, our logs did not include any textual content from participants' accounts.

To help clarify the log data gathered during each session, we administered a questionnaire at the end of each task. In the questionnaire, the participants were asked whether they found relevant 


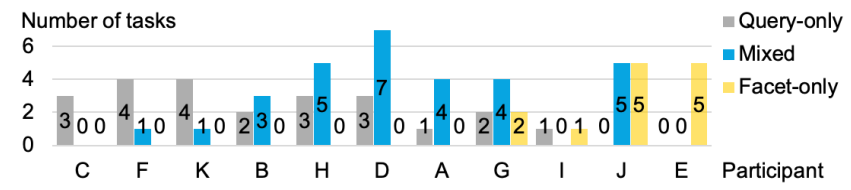

Figure 4: Some participants $(\mathrm{C}, \mathrm{F}, \mathrm{K})$ were more oriented toward typed queries, whereas other participants $(\mathrm{J}, \mathrm{E})$ relied more on timeline navigation and search suggestions.

Table 1: The usage of facets among the 66 search tasks.

\begin{tabular}{rcc}
\hline & $\begin{array}{c}\text { Timeline } \\
\text { (Linear facet) }\end{array}$ & $\begin{array}{c}\text { Suggestions } \\
\text { (Categorical facet) }\end{array}$ \\
\hline Use & $39(59 \%)$ tasks & $38(58 \%)$ tasks \\
Success & $17(26 \%)$ tasks & $23(35 \%)$ tasks \\
\hline
\end{tabular}

contacts or keywords from the suggestions and whether the suggestions supplemented any of the information that was missing at the beginning of the search session.

\subsection{Results}

In total, we obtained 73 session logs. For seven of these tasks, participants remarked that they did not remember which emails their diary entries referred to. Of the remaining 66 tasks, 58 (88\%) were completed successfully, and $8(12 \%)$ were unsuccessful.

Of the 66 tasks, 23 tasks were query-only, i.e. they did not include any facet use. Thirty tasks were mixed sessions in which participants used queries in combination with facets. Most of these sessions $(20 / 30)$ started with queries to reduce the number of emails in the display before participants proceeded to use facets. Thirteen tasks did not involve any use of typed queries. A majority of the tasks in this group (7/13) started with a timeline navigation action, such as clicking on a month, and then relied on suggestions or the timeline to select emails. A Friedman's test showed the differences in using query or facets is not statistically significant among the participants $\left(\chi^{2}(2)=4.05, \mathrm{p}=0.13\right)$. Some participants were more oriented toward submitting typed queries, whereas others relied on timeline navigation and suggestions (Figure 4). Search strategies also varied among tasks that belonged to the same participant, i.e. participants adapted their strategies to different tasks.

The timeline was used in 39 (59\%) sessions (Table 1). In 17 (44\%) of these sessions, specific timeline navigation actions, such as pointing to months and directly selecting dots, led to the selection of the correct email. Selecting emails through timeline navigation was rarely used at the tasks' initial stages, but typically followed query actions or month-navigation actions. Suggestions were used in the majority of search sessions, 38 (58\%) out of the 66 search sessions. In $23(61 \%)$ of these sessions, suggestions led to the selection of the correct email either directly or when used as queries.

Suggestions were generally used following data-specification actions, such as querying or navigating to a month (35/38). A possible explanation is the lack of relevant suggestions at the start of a search session. The log data indeed shows that relevant suggestions (regarding the emails marked as correct by the users) were not encountered as often at the initial stages of the search compared to later stages, i.e. after participants made data-specification actions.
Table 2: The usage of suggestions among the 72 instances.

\begin{tabular}{rccc}
\hline Feature & Direct selection & Drag filtering & Filter-swipe \\
Instances & $48(67 \%)$ & $11(15 \%)$ & $13(18 \%)$ \\
\hline Type & Contact & Keyword & \\
Instances & $63(88 \%)$ & $9(13 \%)$ & \\
\hline
\end{tabular}

The timeline can provide a context to support suggestion discovery. Out of the 72 instances of suggestion usage, $32(44 \%)$ of them were accessed through the timeline, i.e. participants accessed suggestions when hovering over time periods. This reveals that the timeline can provide a context for suggestion exploration. The average time participants spent highlighting suggestions before clicking on them was 1.7 seconds $(\mathrm{SD}=2.1)$. Forty-one of these highlighted actions (57\%) took less than one second, while 18 (25\%) took more than two seconds. Some suggestion selections were preceded by a chain of different suggestions highlighted in sequence.

Suggestions were used more frequently for selection than filtering. Among 72 instances of suggestion use, suggestions were dragged to the query area for filtering only $11(15 \%)$ times (Table 2). A Wilcoxon signed-rank test showed using suggestions for selection was statistically significantly more frequent than for filtering among the participants with a $\mathrm{p}$-value $=0.037$ and an effect size $r=0.645$. In other words, participants generally preferred to keep their current context to inspect items rather than decreasing the size of the item space. Additionally, we collected 13 instances of filter-swipe usage, a small portion (18\%) of the 72 total instances of suggestion usage.

Table 3: Questionnaire results. Contacts were found to be relevant and supplemented missing information more frequently than keyword suggestions. Note that the cases in which the suggestions supplemented missing information are a subset of the cases in which they were found relevant.

\begin{tabular}{rcc}
\hline & Found relevant & $\begin{array}{c}\text { Supplemented } \\
\text { missing information }\end{array}$ \\
\hline $\begin{array}{r}\text { Sender } \\
\text { Co-recipient }\end{array}$ & 30 tasks $(45 \%)$ & 8 tasks $(12 \%)$ \\
Keyword & 18 tasks $(27 \%)$ & 6 tasks $(9 \%)$ \\
\hline
\end{tabular}

The most commonly used suggestions were contacts. Among the 72 instances in which suggestions were used, the overwhelming majority, 63 (88\%), were contact suggestions (Table 2). A Wilcoxon signed rank test showed contacts were statistically significantly more often used than keywords by the participants with a p-value $=0.006$ and an effect size $r=0.847$. Of the 11 instances in which suggestions were used as filters, all were contact suggestions. The subjective evaluation of the suggestions through the questionnaire (Table 3) shows that the contact suggestions were found relevant for 35 search tasks (53\%) and supplemented missing information in 13 tasks (20\%). For keyword suggestions, the respective figures were 18 (27\%) and $11(17 \%)$. A Wilcoxon signed rank test showed participants tend to find contacts more relevant for email finding than keywords with a $p$-value $=0.054$ and an effect size $r=0.585$, which echoed the earlier findings on email search queries that most queries referred to people and especially to senders [17]. 
In summary, the 66 search cases demonstrate that the IVF tool facilitates email finding. A majority (65\%) involved the use of facets to guide searches. Dynamic query suggestions through the timeline navigation can help discover relevant suggestions in the context (R1) in which contact suggestions were more often used than keyword suggestions. The design of using facet values to select items without filtering the item space (R2) was favoured over using facet values as queries to filter the item space among the participants.

\section{DISCUSSION}

The IVF tool, which coordinates a linear facet, a categorical facet, and result items, exemplifies the two design requirements, enabling contextual information and rapid transitions between search criteria, to support fluid exploratory searches. A use case and a user study revealed the usefulness of the tool in supporting exploratory search. The novel design concept of using facet values to select items for inspection without filtering the item space was favoured over using facets as filters according to the user study. Based on the findings, we discuss a set of implications to further address the design requirements and to inspire the design of fluid exploratory searches using IVF.

\subsection{Design implications}

Semantic zooming of the linear facet. To improve user exploration of the contextual information, we could devise the tool to support semantic zooming of the linear facet, such as zooming in or out to visualise bars over the span of days, weeks, and months, then users can aggregate the dots into semantically meaningful collections for pattern recognition. This allows the facet space to hold more items in one view and increase the flexibility of user exploration.

Visual encoding of the item selection order. To enable quick item selection without filtering the item space, the snippet view functions as a stack in which the most recent selections replace older ones. Although this permits flexible switching between selection modes, such as using a suggestion or directly selecting an item from the timeline, it also occasionally makes it harder for participants to recognise the emails that are related to their most recent selections. Currently, we encode item selection order using dot saturation, which is less efficient for visual perceptions compared to position encodings according to Mackinlay's ranking, in which visual variables affect how accurately humans perform the corresponding perceptual task for various data types [25]. A simple solution would be to fix the position of the order of selected items. For example, using a rolling mechanism to always push the newest selected items to the top and move older items toward the bottom can help improve users' sense of item selection.

\subsection{Limitations}

Scalability. The IVF tool's linear facet can display scalable numbers of items, as it arranges linear spans into pages. As discussed earlier, semantic zooming of the linear facet that aggregates the dots into semantically meaningful glyphs can be incorporated to allow one page to hold more items. Regarding the scalability of facets, like most faceted visualisations, the tool is limited in terms of the dimensions of the metadata that it can process.

Generalizability of the user study. The user study did not have a baseline to provide a more quantitative comparison. The participants of the user study were limited to university affiliates and the number was not large, which may hinder the generalizability of the results to a larger population. Moreover, we are interested in whether the findings from the email study can be generalized to other contents such as tweets. More studies are required with other types of content to explore the results' generalizability.

\section{CONCLUSION}

This paper focuses on devising IVF to support fluid interactions for exploratory search. First, we reviewed existing faceted search interfaces by their interaction and visualisation design of facets. We then proposed the idea of IVF and derived two design requirements that have not been well addressed to support fluid, exploratory searches. We exemplified the requirements by devising an IVF tool. The tool comprises an item snippet view, a facet space, and a query field to support multiple entry points for search. The facet space coordinates a linear and a categorical facet to represent the distribution and summarisation of a collection of items and provide contextual information for faceted exploration (R1). The novel design concept of using facets to select items without filtering the item space introduced by the tool facilitates rapid transitions between search attempts (R2). On the one hand, users can quick-select and inspect relevant items by clicking on a categorical facet value; on the other hand, users can employ a filter-swipe technique to dynamically preview results under the flexible combination of two facet values.

We then presented a use case for email finding to illustrate how users could find the contact of interest through the timeline navigation and use the contact for quick item inspection and discovery. We investigated the practical use of the IVF tool through a lab study with realistic email finding tasks. Results show that, often, dynamic suggestions though the timeline navigation helps users discover relevant search suggestions in the context (R1). Among the participants, the design of facet-based item selection that does not filter the item space was favoured over using facet values as queries to filter data (R2). The filter-swipe technique is not ideal for detailed item inspection, but is useful for item skimming.

Based on these practices, we derived a set of design implications to further address the requirements for fluid exploratory searches. First, semantic zooming of the linear facet can further facilitate recognition of data distribution. Second, using position alongside saturation to encode the order of results can improve recognition of recent results. Finally, facet scalability remains a challenge for visualisation. As future work, a user study with a baseline system and more participants of diverse backgrounds could help quantify the findings; studies on other types of content are needed to generalize the findings.

\section{ACKNOWLEDGMENTS}

We thank Heidi Sahlberg from the University of Helsinki for her help with the email user study. This research was supported by the Academy of Finland through the SRC project DataLit.

\section{REFERENCES}

[1] Salvatore Andolina, Khalil Klouche, Tuukka Ruotsalo, Patrik Floréen, and Giulio Jacucci. 2018. QueryTogether: Enabling entity-centric exploration in multi-device collaborative search. Information Processing \& Management 54, 6(2018), 1182-1202.

[2] Kumaripaba Athukorala, Dorota Glowacka, Giulio Jacucci, Antti Oulasvirta, and Jilles Vreeken. 2016. Is exploratory search different? A comparison of information 
search behavior for exploratory and lookup tasks. 7. Assoc. Inf. Sci. Technol. 67, 11 (2016), 2635-2651.

[3] Joseph Chee Chang, Nathan Hahn, Adam Perer, and Aniket Kittur. 2019 SearchLens: Composing and Capturing Complex User Interests for Exploratory Search. In Proceedings of the International Conference on Intelligent User Interfaces. ACM, 498-509.

[4] Siripinyo Chantamunee, Kok Wai Wong, and Chun Che Fung. 2020. An exploration of user-facet interaction in collaborative-based personalized multiple facet selection. Knowl. Based Syst. 209 (2020), 106444.

[5] Cecilia di Sciascio, Peter Brusilovsky, Christoph Trattner, and Eduardo E. Veas. 2020. A Roadmap to User-Controllable Social Exploratory Search. ACM Trans. Interact. Intell. Syst. 10, 1 (2020), 8:1-8:38.

[6] Cecilia di Sciascio, Peter Brusilovsky, and Eduardo E. Veas. 2018. A Study on User-Controllable Social Exploratory Search. In Proceedings of the International Conference on Intelligent User Interfaces. ACM, 353-364.

[7] Cecilia di Sciascio, Vedran Sabol, and Eduardo E. Veas. 2016. Rank As You Go: User-Driven Exploration of Search Results. In Proceedings of the International Conference on Intelligent User Interfaces. ACM, 118-129.

[8] Cecilia di Sciascio, Vedran Sabol, and Eduardo E. Veas. 2017. Supporting Exploratory Search with a Visual User-Driven Approach. ACM Trans. Interact Intell. Syst. 7, 4 (2017), 18:1-18:35.

[9] Cecilia di Sciascio, Eduardo E. Veas, Jordan Barria-Pineda, and Colleen Culley. 2020. Understanding the effects of control and transparency in searching as learning. In Proceedings of the International Conference on Intelligent User Interfaces ACM, 498-509.

[10] Marian Dörk, Sheelagh Carpendale, Christopher Collins, and Carey Williamson 2008. VisGets: Coordinated visualizations for web-based information exploration and discovery. IEEE Trans. Vis. Comput. Graph. 14, 6 (2008), 1205-1212.

[11] Marian Dörk, Sheelagh Carpendale, and Carey Williamson. 2012. Fluid Views: A zoomable search environment. In Proceedings of the International Working Conference on Advanced Visual Interfaces. ACM, 233-240.

[12] Marian Dörk, Daniel Gruen, Carey Williamson, and Sheelagh Carpendale. 2010. A visual backchannel for large-scale events. IEEE Trans. Vis. Comput. Graph. 16, 6 (2010), 1129-1138

[13] Marian Dörk, Nathalie Henry Riche, Gonzalo Ramos, and Susan Dumais. 2012 PivotPaths: Strolling through faceted information spaces. IEEE Trans. Vis. Comput Graph. 18, 12 (2012), 2709-2718.

[14] Niklas Elmqvist, Andrew Vande Moere, Hans-Christian Jetter, Daniel Cernea, Harald Reiterer, and T. J. Jankun-Kelly. 2011. Fluid Interaction for Information Visualization. Information Visualization 10, 4 (Oct. 2011), 327-340.

[15] David Elsweiler, Mark Baillie, and Ian Ruthven. 2008. Exploring Memory in Email Refinding. ACM Transactions on Information Systems (TOIS) 26, 4, Article 21 (Oct 2008), 36 pages.

[16] Hilary J. Grierson, Jonathan R. Corney, and G. D. Hatcher. 2015. Using visual representations for the searching and browsing of large, complex, multimedia data sets. Int. F. Inf. Manag. 35, 2 (2015), 244-252.

[17] Morgan Harvey and David Elsweiler. 2012. Exploring Query Pattern in Email Search. In Proceedings of the European Conference on Advances in Information Retrieval. Springer-Verlag, Berlin, Heidelberg, 25-36. https://doi.org/10.1007/978-3-642-28997-2_3

[18] Marti Hearst, Ame Elliott, Jennifer English, Rashmi Sinha, Kirsten Swearingen, and Ka-Ping Yee. 2002. Finding the flow in web site search. Commun. ACM 45, 9 (2002), $42-49$

[19] T.J.Jankun-Kelly, Kwan-Liu Ma, and Michael Gertz. 2007. A Model and Framework for Visualization Exploration. IEEE Trans. Vis. Comput. Graph. 13, 2 (2007), 357-369.

[20] Khalil Klouche, Tuukka Ruotsalo, Diogo Cabral, Salvatore Andolina, Andrea Bellucci, and Giulio Jacucci. 2015. Designing for Exploratory Search on Touch Devices. In Proceedings of the SIGCHI Conference on Human Factors in Computing Systems. ACM, 4189-4198.

[21] Khalil Klouche, Tuukka Ruotsalo, and Giulio Jacucci. 2018. From Hyperlinks to Hypercues: Entity-Based Affordances for Fluid Information Exploration. In Proceedings of the Conference on Designing Interactive Systems. ACM, 401-411.

[22] Khalil Klouche, Tuukka Ruotsalo, Luana Micallef, Salvatore Andolina, and Giulio Jacucci. 2017. Visual Re-Ranking for Multi-Aspect Information Retrieval. In Proceedings of the 2017 Conference on Conference Human Information Interaction and Retrieval. ACM, 57-66.

[23] Christin Katharina Kreutz, Peter Boesten, Alex Witry, and Ralf Schenkel. 2018. FacetSearch: A Faceted Information Search and Exploration Prototype In Proceedings of the Conference "Lernen, Wissen, Daten, Analysen", Vol. 2191 CEUR-WS.org, 215-226.

[24] Bill Kules and Ben Shneiderman. 2008. Users can change their web search tactics: Design guidelines for categorized overviews. Information Processing \& Management 44, 2 (2008), 463-484.

[25] Jock Mackinlay. 1986. Automating the design of graphical presentations of relational information. ACM Transactions On Graphics (Tog) 5, 2 (1986), 110-141.

[26] Mohammed Najah Mahdi, Abdul Rahim Ahmad, Mohammed Ahmed Subhi, Roslan Ismail, and Qais Saif Qassim. 2020. Visualization in Faceted Search Engine-A Review. In Proceedings of the Conference on Big Data and Analytics. IEEE, 84-89.
[27] Kostas Manioudakis and Yannis Tzitzikas. 2020. Faceted Search with Object Ranking and Answer Size Constraints. ACM Trans. Inf. Syst. 39, 1 (2020), 9:1-9:33.

[28] Gary Marchionini. 2006. Exploratory search: From finding to understanding. Commun. ACM 49, 4 (2006), 41-46.

[29] Noemi Mauro, Liliana Ardissono, and Maurizio Lucenteforte. 2020. Faceted search of heterogeneous geographic information for dynamic map projection. Inf. Process. Manag. 57, 4 (2020), 102257.

[30] Xi Niu, Xiangyu Fan, and Tao Zhang. 2019. Understanding Faceted Search from Data Science and Human Factor Perspectives. ACM Transactions on Information Systems 37, 2, Article 14 (Jan. 2019), 27 pages.

[31] Xi Niu and Bradley M. Hemminger. 2015. Analyzing the interaction patterns in a faceted search interface. f. Assoc. Inf. Sci. Technol. 66, 5 (2015), 1030-1047.

[32] Catherine Plaisant, Ben Shneiderman, Khoa Doan, and Tom Bruns. 1999. Interface and Data Architecture for Query Preview in Networked Information Systems. ACM Trans. Inf. Syst. 17, 3 (1999), 320-341.

[33] Pernilla Qvarfordt, Gene Golovchinsky, Tony Dunnigan, and Elena Agapie. 2013. Looking ahead: Query preview in exploratory search. In The 36th International ACM SIGIR conference on research and development in Information Retrieval. ACM, 243-252.

[34] Juan Ramos. 2003. Using TF-IDF to determine word relevance in document queries. In Proceedings of the instructional conference on machine learning, Vol. 242. Citeseer, 29-48.

[35] Manuela Rauch, Werner Klieber, Ralph Wozelka, Santokh Singh, and Vedran Sabol. 2015. Knowminer Search - A Multi-visualisation Collaborative Approach to Search Result Analysis. In International Conference on Information Visualisation. 379-385. https://doi.org/10.1109/iV.2015.72

[36] Tuukka Ruotsalo, Jaakko Peltonen, Manuel J. A. Eugster, Dorota Glowacka, Patrik Floréen, Petri Myllymäki, Giulio Jacucci, and Samuel Kaski. 2018. Interactive Intent Modeling for Exploratory Search. ACM Trans. Inf. Syst. 36, 4 (2018), 44:1-44:46.

[37] Christin Seifert, Johannes Jurgovsky, and Michael Granitzer. 2014. FacetScape: A Visualization for Exploring the Search Space. In International Conference on Information Visualization. IEEE, 94-101.

[38] Ben Shneiderman, Don Byrd, and W Bruce Croft. 1997. Clarifying search: A user-interface framework for text searches. D-lib magazine 3, 1 (1997), 18-20.

[39] The Stanford Natural Language Processing Group. 2014. Natural Language Processing Software. https://nlp.stanford.edu/software. Accessed in December 2020.

[40] Fernanda B. Viégas, Scott Golder, and Judith Donath. 2006. Visualizing Email Content: Portraying Relationships from Conversational Histories. In Proceedings of the SIGCHI Conference on Human Factors in Computing Systems. ACM, 979-988.

[41] Ryen W White and Resa A Roth. 2009. Exploratory search: Beyond the query-response paradigm. Synthesis lectures on information concepts, retrieval, and services 1,1 (2009), 1-98.

[42] Barbara M. Wildemuth and Luanne Freund. 2012. Assigning Search Tasks Designed to Elicit Exploratory Search Behaviors. In Proceedings of the Symposium on Human-Computer Interaction and Information Retrieval. ACM, Article 4, 10 pages.

[43] William W. Cohen, MLD, CMU. 2015. Enron Email Dataset. http: //www.cs.cmu.edu/ enron. Accessed in December 2020.

[44] Max Wilson, Alistair Russell, Daniel A Smith, et al. 2006. mSpace: Improving information access to multimedia domains with multimodal exploratory search. Commun. ACM 49, 4 (2006), 47-49.

[45] M. A. Yalcin, N. Elmqvist, and B. B. Bederson. 2017. Keshif: Rapid and Expressive Tabular Data Exploration for Novices. IEEE Trans. Vis. Comput. Graph. 24, 8 (2017), 2339-2352.

[46] Guoqiang Zhang, Shiqiang Tao, Ningzhou Zeng, and Licong Cui. 2020. Ontologies as nested facet systems for human-data interaction. Semantic Web 11, 1 (2020), 79-86.

[47] Jian Zhao, Christopher Collins, Fanny Chevalier, and Ravin Balakrishnan. 2013. Interactive Exploration of Implicit and Explicit Relations in Faceted Datasets. IEEE Trans. Vis. Comput. Graph. 19, 12 (2013), 2080-2089. 